\title{
CONTRIBUIÇÃO AO ESTUDO DAS POPULAÇŌES NATURAIS DE Drosophila willistoni DO ESTADO DO RIO GRANDE DO SUL
}

Claudete M.B. de Borba

Departamento de Biologia. Centro de Ciências Naturais e Exatas. UFSM. Santa Maria, RS.

Marly Napp

Departamento de Genética. UFRGS. Caixa Postal 1953. Porto Alegre. RS.

RESUMO

Quarenta e seis amostras de populações nạturais de $D$. wiz Listoni de três localidades do estado do Rio Grande do Sul foram es tudadas entre dezembro de 1978 e julho de 1981. Os dados sugerem que essa espēcie tem o tamanho populacional regulado por uma interação entre temperatura e umidade.

0 coquinho (Arecastrum romanzoffianum) mostrou ser um subs trato adequado tanto para alimentação como para ovoposição.

Entre as localidades estudadas, no Parque Florestal Esta dual do Turvo foi possível observar uma população adequada de $D$. willistoni na maioria dos periodos de coleta, provavelmente devido às condições climāticas e ecolōgicas mais favorāveis para a espécie nesse local.

SUMMARY

BORBA, C.M.B. de. and NAPP, M. 1985. Contribution to the study of natural populations of Drosophila wizlistoni of the Rio Grande do Sul State. Ciência e Natura, 7: 181 - 195.

Forty six samples of natural populations of D. wizlistoni from three places of Rio Grande do Sul state were studied between december 1978 and july 1981. The data sugest that this species have a regulation of populacional size by a interection between temperature and humidity.

A small coconut (Arecastrum romanzoffianum) show to be a suitable substrate to nurture and also to ovoposition.

Among the places studied,at the Parque Florestal Estadual do Turvo it was possible to observe a fit population of $D$. wizlisto ni in majority of gathering periods, probabily because the ecological and climatic conditions were move benefic at this place than at the two others.

INTRODUÇÃO

Uma vez detectada a variabilidade genética que existe de forma ampla e generalizada nas populações naturais (Hubby e Lewontin, 
1966; Johnson e cols. 1966), diversos autores têm se preocupado em realizar trabalhos que explicitem os fatores que originam e mantém tal variabilidade. o gēnero Drosophila têm sido usado, extensiva e intensivamente, na busca da resposta a esse problema. Entretanto vá rias conclusões que foram obtidas atravēs de estudos com populações experimentais têm sido objeto de questionamento, baseado no argumen to de que foram resultado de dados de laboratório, onde as condi ções ambientais são. obviamente diferentes das que ocorrem na nature $z a$. Daî porque a grande ênfase que se tem proporcionado a estudos so bre as condições ecológicas a que estão submetidas as populações na turais de Drosophila para, só então, poder concluir sobre o modo co mo a constituição genética da população se comporta no curso da evo lução.

Para tanto, é preciso considerar a distribuição espacial da espécie em três níveis diferentes, conforme salientado por Pit tendrigh (1958, citado por Winge, 1971):

10) macrodistribuição ou distribuição geogrāfica;

20) mesodistribuição, que seria o tipo de habitat em que as espécies são mais comumente encontradas;

30) microdistribuição,que se refere ao nicho ecológico ocupada pela espécie.

No Brasil, Dobzhansky e Pavan (1950) estudaram espécies de Drosophila de dezessete regiões bioclimáticas do Brasil e verifica ram que algumas como D. willistoni, D. nebulosa e D. paulistorum apresentavam grande versatilidade ecológica, enquanto outras eram comuns apenas em determinados ambientes ou estações do ano, como $D$. prosaltans e $D$. bandeirantorum, entre outras.

No estado do Rio Grande do Sul, coletas realizadas por vá rios pesquisadores, em diversas localidades, revelaram a $D$. wizlis toni como uma das espécies mais abundantes, quer nas coletas feitas com rede sobre iscas convencionais de banana ou sobre iscas naturais, quer coletando frutos fermentados e após, os imagos que emergiam des ses (Petersen 1960, Brncic e Valente, 1978, Franck, 1982).

Conforme salientado por Toda (1973 b), para que se possa entender a real microdistribuição das populações naturais de droso filīdeos é necessārio obter ampla informação sobre os häbitos de alimentação e cruzamento nas condições naturais. Para is to se requer estudos que envolvam a aplicação de vārios métodos de amostragem, tais como vários tipos de iscas naturais, junto com as iscas conven cionais introduzidas no local. As observações de Dudgeon (1954) e da Cunha (1957), são consistentes com a hipōtese de que a maioria das drosófilas são altamente oportunistas em relação a sítios de alimentação, utilizando como instrumentos de busca desses sîtios 
seus sentidos olfativos altamente desenvolvidos e seus consideráveis poderes de vôo. Por outro lado, seria vantajoso que a espēcie não fosse rïgida em relação a suas preferēncias alimentares, de modo que, em caso de faltar o alimento preferido, ela pudesse subsistir por algum tempo com a utilização de outro recurso (da Cunha, 1951). Jā a ovoposição é, para a maioria das espécies de Drosophita, uma performance mais especializada, tanto que quando a ovoposição ocor re em um sitio, esse deixa de ser usado como recurso alimentar, sen do utilizado apenas para ovopositar. (Carson, 1971). Kaneshiro e cols. (1973) mostraram que o comportamento de ovoposição é um fa tor essencial na manutenção e separação de nicho em um par de espé cies simpātricas de Drosophiza da ilha de Hawaii.

Entretanto é difícil estabelecer a correlação dos sîtios de alimentação e ovoposição, como foi assinalado por Heed (1957) que, coletando espécies de Drosophiza em El Salvador, observou que das quatro espécies que emergiram de uma isca natural apenas $D$. succinea havia sido coletada com rede quando sobrevoava essa mesma isca.

Carson (1965) obteve informações sobre sîtios de cruzamen to de dezessete espécies amplamente distribuĩdas, observando que a maioria tem sido encontrada cruzando em uma variedade de substratos. Inclui-se aî a $D$. wizlistoni que, alēm do cruzamento sobre frutos maduros caídos ao solo, como observado por Heed (1957), deve utili zar tambēm outros sītios de cruzamento, jā que Birch e Battaglia (1957) observaram, em algumas ilhas da costa do Brasil, que a especie era comum mesmo na ausència de frutos.

Embora adultos e larvas de Drosophiza sejam micófagos, a grande maioria das espécies utilizando substâncias vegetais em fer mentação, as diferenças nutricionais entre as espécies constituem-se numa das principais fontes de diversificação dos habitats (Pavan, 1952). Foi verificado que uma mesma espécie pode ser encontrada vi vendo sobre um tipo de fruta em uma região, não o sendo em outra, 0 que sugere uma diversificação racial da espécie (Winge, 1971). A comprovação da seleção de habitat como um mecanismo generalizado na natureza é de fundamental importância na determinação da estrutura genētica das populações, uma vez que permitiria uma ampla heteroge neidade intrapopulacional, enfatizando assim o papel das populações locais como a unidade da seleção (Parsons e Stanley, 1981).

Respostas comportamentais a extremos de temperatura e umi dade foram encontrados por Parsons (1975) em D. inornata e por Ar lan e Eckstrand (1975) em D. pseudoobscura, sendo possivel que a maioria das espēcies de Drosophiza respondam similarmente. As mos cas dispersam-se uniformemente na floresta quando a umidade $\bar{e}$ alta, mas movem-se em direção à àgua nos perĩodos de alta tamperatura ou baixa umidade. 0 limite inferior de temperatura suportado estā 
provavelmente associado com o limite inferior de degradação micro biana efetiva de vegetais, necessária para o envolvimento de Droso phiza (Parsonș e Stanley, 1981).

Como salientado por Pavan (1959) a condição mais adequada para o organismo é o desenvolvimento de um comportamento não rĩgi do, que permita à espécie coexistir com as demais que exploram os mesmos recursos, preferindo condições que 1 he sejam mais favoráveis, mas podendo sobreviver em condições outras. A seleção natural como um processo oportunista, apresenta uma solução para cada caso, seja sob forma de luta, de cooperação de vārios tipos, de qualquer rela ção que convenha à espēcie na época, independentemente do que irā acontecer no futuro.

\section{MATERIAL E METODOS}

Foram estudadas populações naturais da espécie Drosophiza wizzistoni Sturtevant dos seguintes locais:

a) Estação Experimental Agronōmica da Universidade Fede ral do Rio Grande do Sul (EEA-UFRGS) - município de Guaíba, RS. Ló cal situado a $40 \mathrm{~km}$ de Porto Alegre, na direção oeste, pertencendo à região fisiogrāfica da Depressão Central (Fortes, 1959). Seu tipo de formação vegetal ê o dos chamados "capões", trecho de mato arre dondado e fechado que se destaca nitidamente na paisagem de campo que o circunda, e que Rambo (1956) considerou como uma mata virgem em escala natural que parece haver sido artificialmente podada.

b) Itapuã - município de Viamão, RS. Situa-se cerca de 60 Km ao Sul de Porto Alegre e pertence à região fisiográfica da Depres são Central. (Fortes, 1959). o local possui uma extensa mata onde predominam arbustos e ārvores de pequeno porte, embora ocorram algu mas ārvores de maior porte, como as figueiras. Saàlfeld (1979)obser va que a umidade relativa do ar parece ser bastante alta no local, devido à proximidade da Lagoa Negra e à ocorrência de vertentes na encosta do morro, o que contrabalançaria a grande permeabilidade do solo arenoso.

Devido à extensão do local, este foi subdividido arbitra riamente pelo grupo de pesquisadores que a ī trabalharam, em āreas que receberam um nūmero. A ārea em que nossas coletas foram realiza das foi a denominada Area 2, citada por Saalfeld (1979) e Albuquer que (1979).

C) Parque Florestal Estadual do Turvo, município de Tenen te Portela, RS. Situado na região fisiogräfica do Alto Uruguai (Fortes, 1959) localiza-se cerca de $500 \mathrm{~km}$ ao norte de Porto Alegre. A área ē classificada como mata pluvial subtropical perenifólia do Alto Uruguai, conforme Irgang (1980). 0 mesmo autor refere que essa mata apresenta, de maneira geral, 0 estrato arbōreo superior, de ārvores de 30 a $40 \mathrm{~m}$., o estrato médio e 
- estrato arbōreo baixo, com ärvores pequenas, além dos estratos ar bustivo e herbāceo.

As coletas foram feitas ao longo de duas estradas inter nas do Parque: a estrada do Porto Garcia que desce em direção ao Rio Uruguai, numa extensão de $9 \mathrm{Km}$., e a estrada do Salto que se di rige, com $16 \mathrm{Km}$ de extensão, ao Salto Yacumã, no Rio Uruguai. Como a estrada do Porto é mais extensa e fechada que a do Salto, o am biente na primeira mantēm-se ūmido e sombrio, enquanto na segunda apresenta-se seco e ensolarado., conforme observado por Hofman (1982).

Nos três locais as drosōfilas foram coletadas sobre iscas convencionais de banana (Musa paradisiaca), com o auxîlio de uma re de, ao mesmo tempo em que eram apanhados frutos caídos ao solo, com um certo grau de fermentação natural. A técnica usada para a coleta foi a descrita por Brncic e Valente (1978).

Os frutos coletados foram trazidos em sacos plásticos atē - laboratōrio de Genētica da UFRGS e aî distribuīdos em tubos de vi dro com meio de cultura usado rotineiramente (Marques e cols., 1966), sendo então colocados numa câmara a $25^{\circ} \mathrm{C}$ até a emergēncia dos imagos, a partir dos ovos e larvas localizados no interior dos frutos. Após, procedia-se a separação, por espécie, dos indivĩduos que haviam emer gido no laboratório, e dos que haviam sido coletados sobre as iscas naturais e sobre as iscas introduzidas no local de coleta.

\section{RESULTADOS}

Itapuã

Nas coletas realizadas observou-se uma frequência menor de $D$. wizzistoni do que o total das demais espëcies do gênero, com exceção da coleta feita sobre isca de banana em maio de 1980 quando aquela espēcie atingiu a frequência de 0,5189. Já na amostra coleta da sobre a mesma isca em novembro de 1979, verificou-se a menor fre quência da $D$. wizlistoni em todas as coletas, jā que nessa ocasião constituiu apenas 0,0043 do total da amostra. (Tabela I).

Quanto às diversas iscas utilizadas observou-se a prefe rência pela isca convencional de banana introduzida no local. Entre as iscas naturais foi o coquinho (Arecastrum romanzoffianum) a pre ferida, tanto para cruzamento e/ou alimentação dos adultos (iscas designadas como(Coq. (sobre)), quanto para ovoposição e consequlente desenvolvimento das larvas (iscas Coq. (dentro)). A Tabela I apre senta um resumo dos dados de coleta.

Estação Experimental Agronômica - UFRGS

No período em que foi iniciado o trabalho de coleta nesse local, observou-se uma desvantagem numērica da $D$. wizzistoni, em re lação às demais espécies do gênero, desvantagem esta que se acentou progressivamente, de junho/79 a outubro do mesmo ano. Esta situação 
foi revertida a partir dessa época, de tal modo que em março de 1980 a D. willistoni atingiu uma frequência de 0,5300 do total de drosó filas coletadas. (Tabela II).

TABELA I. COLETAS REALIZADAS EM ITAPUA, RS.

\begin{tabular}{|c|c|c|c|c|}
\hline Isca & Epoca & $\begin{array}{l}\text { Freqdência de } \\
\text { D. Wilzistoni }\end{array}$ & $\begin{array}{l}\text { Frequência de } \\
\text { outras espécies }\end{array}$ & Total \\
\hline Banana & $12 / 78$ & 0,2028 & 0,7972 & 493 \\
\hline Banana & $01 / 79$ & 0,0614 & 0,9386 & 29.551 \\
\hline Banana & $08 / 79$ & 0,0196 & 0,9804 & 4.395 \\
\hline Banana & $10 / 79$ & 0,0108 & 0,9892 & 743 \\
\hline Banana & $11 / 79$ & 0,0043 & 0,9957 & 5.388 \\
\hline Banana & $12 / 79$ & 0,0255 & 0,9745 & 4.905 \\
\hline Banana & $03 / 80$ & 0,2370 & 0,7630 & 4.434 \\
\hline Banana & $05 / 80$ & 0,5189 & 0,4811 & 2.625 \\
\hline Banana & $05 / 81$ & 0,1022 & 0,8978 & 2.299 \\
\hline Coq. (sobre) & $05 / 81$ & 0,3640 & 0,6360 & 544 \\
\hline Coq. (dentro) & $05 / 81$ & 0,2019 & 0,7981 & 2.987 \\
\hline Banana & $07 / 81$ & 0,2584 & 0,7416 & 2.516 \\
\hline $\begin{array}{c}\text { Jacu macho } \\
\text { (sobre) }\end{array}$ & $07 / 81$ & 0,3427 & 0,6573 & 429 \\
\hline $\begin{array}{l}\text { Jacu macho } \\
\text { (dentro) }\end{array}$ & $07 / 81$ & 0,6328 & 0,3672 & 207 \\
\hline
\end{tabular}

TABELA II. COLETAS REALIZADAS NA ESTAÇĀO EXPERIMENTAL AGRONŌMICA, UFRGS, EM GUAIBA, RS.

\begin{tabular}{lcccr}
\hline \multicolumn{1}{c}{ Isca } & Epoca & $\begin{array}{c}\text { Frequência de } \\
\text { D. wizlistoni }\end{array}$ & $\begin{array}{c}\text { Frequência de } \\
\text { outras espécies }\end{array}$ & Total \\
\hline Banana & $06 / 79$ & 0,1130 & 0,8870 & 2.017 \\
Banana & $07 / 79$ & 0,0304 & 0,9696 & 1.185 \\
Banana & $08 / 79$ & 0,0045 & 0,9955 & 2.240 \\
Banana & $09 / 79$ & 0,0028 & 0,9972 & 14.368 \\
Coq. (dentro) & $09 / 79$ & 0,0526 & 0,9474 & 57 \\
Banana & $10 / 79$ & 0,0026 & 0,9974 & 7.310 \\
Coq. (dentro) & $10 / 79$ & 0,0704 & 0,9296 & 1.066 \\
Banana & $11 / 79$ & 0,0446 & 0,9554 & 5.154 \\
Coq. (sobre) & $11 / 79$ & 0,1991 & 0,8009 & 1.798 \\
Coq. (dentro) & $11 / 79$ & 0,2733 & 0,7267 & 2.272 \\
Banana & $12 / 79$ & 0,4214 & 0,4547 & 2.411 \\
Coq. (sobre) & $12 / 79$ & 0,5453 & 0,2268 & 2.241 \\
Coq. (dentro) & $12 / 79$ & 0,7732 & 0,4667 & 895 \\
Banana & $03 / 80$ & 0,5333 & 0,5131 & 1.635 \\
Banana & $04 / 80$ & 0,4869 & 0,7310 & 3.744 \\
Banana & $01 / 81$ & 0,2690 & 0,7540 & 3.346 \\
Banana & $05 / 81$ & 0,2460 & 0,7610 & 159 \\
Coq. (dentro) & $05 / 81$ & 0,2390 & & \\
\hline
\end{tabular}

observou-se aqui, como nas coletas realizadas em Itapuã, a nītida preferência da espécie pela isca natural "coquinho"(Arecastmon 
romanzoffianum) como fonte de alimentação/ovoposição. Esta preferēn cia superou inclusive a isca convencional de banana, pois, enquanto esta atraiu uma quantidade de $D$. willistoni equivalente a 0,446 do total de drosófilas coletadas, na coleta feita sobre o coquinho o percentual da citada espécie atingiu 0,991 do total.

Em dezembro de 1979 a isca coquinho mostrou ser a mais atrativa tambēm para ovoposição, jā que, do total de drosófilas que emergiu dos frutos coletados ao solo, 0,7732 pertenciam à espécie D. willistoni.

Parque Florestal e Estadual do Turvo

Nas amostras coletadas sobre as iscas, tanto as naturais como as introduzidas no local, observou-se um nîtido predomínio da D. wizlistoni em relação às demais espécies do gênero, já que atin giu uma frequência de, pelo menos 0,5000 , como evidenciado pela Ta bela III. A única exceção verificou-se na amostra coletada sobre a isca convencional de banana, no inverno de 1980 , quando sua frequên cia foi de apenas 0,0650 de todas as drosófilas coletadas na época.

TABELA III. COLETAS REALIZADAS NO PARQUE FLORESTAL ESTADUAL DO TURVO.

\begin{tabular}{|c|c|c|c|c|}
\hline Isca & Epoca & $\begin{array}{l}\text { Frequência de } \\
\text { D. willistoni }\end{array}$ & $\begin{array}{l}\text { Frequēncia de } \\
\text { outras espécies }\end{array}$ & Total \\
\hline $\begin{array}{l}\text { Banana } \\
\text { Banana }\end{array}$ & $\begin{array}{l}01 / 80 \\
04 / 80\end{array}$ & $\begin{array}{l}0,4967 \\
0,8760\end{array}$ & $\begin{array}{l}0,5033 \\
0,1240\end{array}$ & $\begin{array}{l}7.151 \\
1.709\end{array}$ \\
\hline $\begin{array}{l}\text { Coquinho } \\
\text { (sobre) P }\end{array}$ & $04 / 80$ & 0,6860 & 0,3140 & 777 \\
\hline $\begin{array}{l}\text { Coquinho } \\
\text { (sobre) P }\end{array}$ & $04 / 80$ & 0,9583 & 0,0417 & 575 \\
\hline $\begin{array}{l}\text { Coquinho } \\
\text { (sobre) S }\end{array}$ & $04 / 80$ & 0,6888 & 0,3112 & 980 \\
\hline $\begin{array}{l}\text { Coquinho } \\
\text { (dentro) } \mathrm{s}\end{array}$ & $04 / 80$ & 0,4951 & 0,5049 & 507 \\
\hline $\begin{array}{l}\text { Banana } \\
\text { Banana }\end{array}$ & $\begin{array}{l}07 / 80 \\
02 / 81\end{array}$ & $\begin{array}{l}0,0650 \\
0,7766\end{array}$ & $\begin{array}{l}0,9350 \\
0,2234\end{array}$ & $\begin{array}{l}5.602 \\
3.044\end{array}$ \\
\hline $\begin{array}{l}\text { Sete Capotes } \\
\text { (sobre) }\end{array}$ & $02 / 81$ & 0,8077 & 0,1923 & 4.399 \\
\hline $\begin{array}{l}\text { Sete Capotes } \\
\text { (dentro) }\end{array}$ & $02 / 81$ & 0,6263 & 0,3737 & 297 \\
\hline Aguaî (sobre) & $02 / 81$ & 0,7613 & 0,2387 & 1.278 \\
\hline $\begin{array}{l}\text { Aguaî (dentro) } \\
\text { Banana }\end{array}$ & $\begin{array}{l}02 / 81 \\
04 / 81\end{array}$ & $\begin{array}{l}0,3846 \\
0,5052\end{array}$ & $\begin{array}{l}0,6154 \\
0,4948\end{array}$ & $\begin{array}{r}104 \\
2.191\end{array}$ \\
\hline $\begin{array}{l}\text { Coquinho S } \\
\text { (sobre) }\end{array}$ & $04 / 81$ & 0,5160 & 0,4840 & 250 \\
\hline
\end{tabular}

Entre as iscas naturais a que atraiu um número maior de willistoni foi Sete-Capotes (Britoa sellowiana), onde essa espécié atingiu uma frequência de 0,8068 . Foi também dessa fruta que emer giu o maior número de adultos da espécie, o que indica ser a is ca um sỉtio adequado para alimentação e ovoposiçãr

outro fato notável foi a atratividade diferencial evidenciada 
pela mesma isca, em diferentes locais. Assim, dos coquinhos coleta dos no Salto em abril de 1980, emergiram 0,4951 indivíduos da espé cie D. wizzistoni em relação ao total de drosöfilas coletadas, en quanto que, na coleta realizada no Porto, este valor ascendeu a 0,9583. Tal diferença torna-se ainda mais marcante quando se observa que o nümero relativo de indivíduos da espēcie em questão foi praticamen te o mesmo nas coletas realizadas sobre as citadas iscas.

\section{DISCUSSÃO}

Nas coletas que realizamos em Itapuã, em 1979, foi nos meses mais quentes que a população de $D$. wizzistoni atingiu suas frequên cias mais baixas, ao contrārio do afirmado por Patterson e Wagner (1943) de que é nos meses de inverno, quando ocorrem baixas tempera turas, que as populações de Drosophizamostram a maior queda nas suas frequencias. Provavelmente o que se observou em nossas coletas foi devido às condições climäticas que ocorreram no mês de janeiro de 1979, quando a precipitação pluviométrica foi de apenas 27,5 mm., pa ra uma temperatura mëdia de $23,2^{\circ} \mathrm{C}$.

Dobzhansky e Pavan (1950) jā haviam constatado ser a umi dade um fator limitante para moscas do grupo wizlistoni, o que foi confirmado por Sene et alii (1980). Ainda em 1979, a partir do mês de agosto, em Itapuã, houve uma alternāncia de dias frios com ou tros já bastante quentes, o que provavelmente tenha causado as al terações que se verificaram no perīodo de frutificação das espécies de plantas utilizadas pela D. wizlistoni para alimentação e/ou ovo posição. A importāncia da quantidade de chuva e dos perīodos de fru tificação das plantas sobre o nūmero de drosōfilas foi mostrado por Mather (1956), coletando na região de Queensland (Austrāiia). Pa van (1959) tambēm abordou a influēncia das condições climáticas só bre a flora de levedos e bactérias que se constitui no principal re curso alimentar das drosófilas. Entretanto, esta estreita relação entre abundância de frutos e da D. wizlistoni não foi verificada por Birch e Battaglia (1957) que observaram que a espécie era comum, mesmo na ausência de frutos.

Nas primeiras coletas realizadas em Itapuã no ano seguin te $(3 / 80$ e 5/80) observou-se um crescimento da população de $D$. wizzis toni, surgindo então a dūvida sobre o que teria acontecido à espé cie durante o período em que sua frequência caiu bastante. Patterson e Wagner (1943) afirmam que quando uma espécie não é encontrada em algum periodo é porque a densidade da população tornou-se tão baixa que os espēcimens não são atraỉdos pelas iscas. Entretanto, para con siderar que a espécie estivesse praticamente ausente da região nos períodos em que se coletou poucos indivíduos, teríamos que supor a sua reintrodução por migração a partir de outras áreas nos perīodos 
anteriores àqueles onde se constatou um aumento na frequência popu lacional. Estudos feitos com populações brasileiras de $D$. wizlisto ni por Burla et alii (1950) mostraram que a espécie apresenta movi mentos de muito curta amplitude, o que os autores atribuem às altas densidades populacionais apresentadas pela espécie, como decorrente de sua grande versatilidade ecológica. Johnston e Heed(1976), com base no observado em $D$. nigrospiracula, sugerem que a taxa de dis persão seria uma resposta evolutiva às condiçoes do habitat, aumen tando nas condiçoes adversas, principalmente estresse alimentar.

Diversos outros autores propõem a hibernação como uma ex plicação provável para os períodos de baixa densidade populacional, quando suficientes espëcimens podem sobreviver como imagos hibernan tes ou como larvas, para assegurar que seja atingida a próxima esta ção regular de cruzamento. (Patterson e Wagner, 1943; Carson e Stal ker, 1948; Toda e Kimura, 1978).

A alta frequência de $D$. wizlistoni observada em maio de 1980 em Itapuã, na coleta feita sobre isca de banana, é uma provāvel consequência das condições apropriadas de temperatura (média de $\left.22,1^{\circ} \mathrm{C}\right)$ e precipitação pluviométrica $(124,5 \mathrm{~mm})$ no mês que antece deu a coleta. Essa observação ratifica mais uma vez a exigēncia da espēcie por um clima quente e úmido para o crescimento de sua popu 1 ação.

Na EEA-UFRGS, a ūnica fruta coletada foi o coquinho (Are castrum romanzoffianum), que demonstrou ser um substrato adequado tan to para adultos como para larvas, uma vez que a frequéncia de $D$. wizlistoni coletada sobre o coquinho, ou dele emergindo, foi sem pre maior do que nas amostras apanhadas sobre a banana. A preferén. cia da D. wizlistoni por esse.fruto jā havia sido assinalada nas co letas realizadas por Birch e Battaglia (1957).

Nas coletas feitas em dezembro de 1979 sobre isca de bana na, a D. wizlistoni atingiu a frequência de 0,0255 em Itapuã e 0,4214 na EEA/UFRGS. Assim, em nenhum dos dois locais foi a espécie mais frequente, como havia sido relatado por Peterson (1960) nas coletas que realizou em dezembro de 1959, na mesma região fisiogrāfica (De pressão Central). Provavelmente essa diferença na frequência da $D$. wizlistoni nas duas épocas citadas (12/59 e 12/79) seja devida à al teração na composição dos recursos ou na eficiência com que os mes mos podem ser utilizados pelas drosōfilas, como resultado das varia ções temporais no clima e outras características ambientais.

Em março de 1980 , as coletas feitas sobre isca de banana introduzida no local evidenciaram diferentes frequéncias em Itapuã $(0,2370)$ e na EEA/UFRGS $(0,5333)$. Como os dois locais situam-se na mesma região fisiogräfica, è de supor-se que haja diferenças na flo ra microbiana dos dois locais, com relação à sensibilidade às variações 
climáticas. Mrak e Phaff (1948, citados por Pavan, 1959) jā hạ viam demonstrado que as espēcies de levedos são muito sensiveis às mudanças ambientais e têm nîtida preferência por habitats definidos.

A grande quantidade de D. wizlistoni observada em quase todos os periodos de coleta no P.F.E. do Turvo, apenas confirmou o observado por Araújo e Valente (1981), que atribuiram o fato à loca lização da ārea em questão numa zona onde ocorrem temperaturas mé dias superiores às das áreas vizinhas mesmo no inverno, e umidade relativa do ar também bastante elevada. Outro fator que favorece a abundāncia da espécie é a variabilidade de recursos oferecidos pelo ambiente. Já que os recursos alimentares utilizados pelasdrosófilas (fungos e bactērias) não são mōveis, pode ser mais vantajoso a in clusão de vārios recursos trōficos na sua dieta, especializando-se na prospecção dos mesmos, como sugerido por Mac Arthur e Pianka (1966). Jaenike (1978) também jā salientava que a polifagia dos indivĩduos é provavelmente favorecida pela seleção, ao mesmo tempo que haveria uma seleção contrária à especialização quanto ao sîtio de ovoposição, sendo mais vantajosa a flexibilidade desse comportamento.

Ainda no P.F.E. do Turvo, em abril de 1980, nas amostras coletadas sobre o coquinho, observamos uma frequéncia praticamente igual, tanto no Porto como no Salto. Entretanto, emergiram menos adultos dos coquinhos coletados ao pé da planta-mãe no Salto(0,4951), do que nas amostras obtida dos coquinhos no porto $(0,9583)$. Isso pa rece evidenciar que ambos os locais foram igualmente adequados para os adultos em busca de recursos alimentares, sendo, porëm, os co quinhos do Porto um sitio preferencial para ovoposição. Carson (1951) chama a atenção para o fato de que cada recurso utilizado para al $\underline{i}$ mentação/ovoposição tem a sua prōpria ontogenia microbiolōgica. As sim, a chegada de um dado microorganismo pode permitir que o sub $\underline{s}$ trato se torne atrativo para moscas em alimentação, apenas mais tar de alcançando uma condição adequada para ovoposição. Quando isso ocorre, a fase de alimentação indiscriminada cessa e o sitio passa a ser usado apenas para ovopositar. Phaff e cols. (1956) afirmam que a divergência nos hábitos alimentares de adultos e larvas seria man tida e aperfeiçoada, visando a redução da competição intra-especīf $\underline{i}$ ca. Carson e cols. (1956) tambēm observaram notāveis diferenças en tre os fungos dos sîtios de criação e de alimentação, como eviden ciado pelas anâlises nos levedos encontrados nas larvas e adultos de uma mesma espēcie.

No presente trabalho, a coincidēncia observada entre os sitios explorados pelos adultos e larvas é consequéncia da metodolo gia escolhida. Como nos interessava comparar amostras populacionais coletadas sobre e dentro dos frutos, propositadamente rejeitamos aque las amostras onde só tínhamos obtido adultos sobrevoando os frutos, 
e nenhuma mosca emergira dos frutinhos fermentados trazidos ao labo ratōrio. Entretanto, a não coincidēncia entre os sîtios de alimenta ção e ovoposição foi observada por vārios autores, como Heed (1957), Araūjo e Valente (1981), Saavedra e Napp (1982). Isso denota, con forme Heed (1957), que o registro obtido das micropopulações é pro vavelmente incompleto, pela dificuldade de estabelecer correlação entre os sítios de alimentação e ovoposição, entre outros fatores.

Conforme observamos em todas as coletas realizadas, as

larvas de $D$. wizlistoni compartilhavam o substrato com larvas de ou tras espécies. Diversos trabalhos tēm sido feitos na tentativa de explicar como seria feita a partilha dos recursos limitados, visan do a minimizar a competição, o que è particularmente crítico na fa se larval, quando è máxima a utilização de recursos (Parsons, 1977). Ayala (1970) afirmou que espécies competidoras podem coexistir em um equilíbrio globalmente estável se elas usam recursos potencial mente diferentes, ou se os utilizam com diferentes eficácias. Hoe nigsberg et alii (1977) observaram membros do grupo wizlistoni em três diferentes ecossistemas, na colómbia, e constataram que existe uma clara seleção temporal, considerando a hora do dia em que maior nümero de drosófilas procuram a isca, o que possibilita a explora ção simpātrica dos mesmos nichos ecológicos.

\section{CONCLUSOES}

Os dados obtidos apontam na direção do seguinte:

1 - As menores frequências relativas da $D$. wizlistoni fo ram obtidas em períodos que, apesar de bastante quentes, foram ex tremamente secos. Isto confirma observações feitas por outros auto res de que a espēcie tem seu tamanho populacional controlado pela interação de temperatura e umidade, sendo os melhores períodos para coleta aqueles em que os dias se apresentam quentes e úmidos.

2- 0 coquinho (Arecastrum romanzoffianum) demonstrou ser um substrato adequado tanto para alimentação quanto para ovoposição da D. wizzistoni, nos três locais de coleta. Esse resultado deve-se ao fato de que sempre foi obtido um nūmero expressivo de indivĩduos dessa espécie entre as drosōfilas que sobrevoavam os frutos caỉdos ao pé da ārvore-mãe, bem como entre as que emergiam desses mesmos fru tos.

3- Locais pertencentes à mesma região fisiográfica (Itapuã e EEA/UFRGS) exibiram diferentes curvas de crescimento populacional para D. wizzistoni, provavelmente devidos a diferenças apresentadas pelas floras microbianas estabelecidas em cada local, em relação à sensibilidade às variações climáticas.

4- Dos três locais estudados, foi no P.F.E. do Turvo que se observou uma razoāvel quantidade de $D$. wizlistoni em quase todos 
os períodos de coleta. Tal fato deveu-se, provavelmente, às condi ções climáticas (temperaturas médias superiores às das āreas vizi nhas, inclusive no inverno e alta umidade relativa do ar) e ecológ $\underline{i}$ cas (ampla variabilidade de recursos para alimentação e/ou ovopos ção) mais favorāveis para a espécie neste local.

5- Quando foram coletadas moscas sobre e dentro de um de terminado fruto, em metade dos casos foi maior o número de moscas apanhadas com rede sobre os frutos caĩdos ao chão do que o daqueles que emergiram dos frutos a $\bar{T}$ coletados, enquanto que, na outra meta de, observou-se a situação inversa. Isso sugere que determinados re cursos são mais utilizados pelos adultos para alimentação enquanto outros são, preferencialmente, aproveitados para ovoposição por mos cas que não permanecem sobrevoando o local, após a postura.

\section{AGRADECIMENTOS}

Nosso agradecimento às instituições e örgãos que subven cionaram este trabalho (CNPq, PIG II e III e CEPP-UFRGS) e às pes soas que conosco colaboraram, especialmente ao Sr. Danúbio Ramila, ao acadêmico Joicemar T. Amaro e ao Prof. Renato Z. Flores.

\section{B I BL I OGRAF I A}

ALBUQUERQUE, C.M.R. 1979. Variabilidade do loco da Esterase-6 em populações naturais de Drosophiza simuzans e sua associação com he terogeneidade ambiental. Dissertação de Mestrado. Curso de PósGraduação em Genētica. Universidade Federal do Rio Grande do Sul. Porto Alegre.

AYALA, F.J. 1970. Competition, coexistence and evolution. In:Eassays in Evolution and Genetics in honor of Theodosius Dobzhansky. p. 121. Ed. M.K. Hecht and W.C. Steere. Appleton Century Crofts. New York.

ARAJjo, A.M. de \& VALENTE, V.L.S. 1981. Observações sobre alguns le pidopteros e drosofilídeos do Parque do Turvo, RS. Ciência e Cul tura, 33(11): 1485-1490.

ARLAN, L.G. and ECKSTRAND, I.A. 1975. Water balance in Drosophiza pseudoobscura, and its ecological implications. Ann. Ent. Sec. U.S. $68: 827-832$.

BIRCH, L.C. \& BATTAGLIA, B. 1957. The abundance of Drosophiza wizlis toni in relation to food in natural populations. Ecology, 38 (1). $165-166$.

BRNCIC, D. \& VALENTE, V.L.S. 1978. Dinämica das comunidades de Dro sophiza que se estabelecem em frutos silvestres no Rio Grande do Su1. Ciēncia e Cultura, 30(9): 1104-1111.

BURLA, H.A.; DA CUNHA, A.B.; CAVALVANTI, A.G.L.; DOBZHANSKY, Th. \& PAVAN, C. 1950. Population density and dispersal rates in Brazilian 
Drosophila willistoni. Ecology, 31: 393-404.

CARSON, H.L. 1951. Breeding sites of D. pseudoobscura and D. persi milis in the transition zone of the Sierra Nevada. Evolution, 5: $91-96$.

CARSON, H.L. 1965. Chromosomal morphism in geographically widespread species of Drosophiza. In: The Genetics of Colonizing Species. Ed. H.C. Baker and G.L. Stebbins. New York, Academic Press. p. 503-531.

CARSON, H.L. 1971. The ecology of Drosophiza breeding sites. Harold L. Lyon Arboretum. Lecture, Uni.Hawaii, 2: 1-28.

CARSON, H.L. \& STALKER, H.D. 1948. Reproductive diapause in Droso phiza robusta. Proc. Nat1. Acad. Sci., USA, 34: 124-129.

CARSON, H.L.; KNAPP, E.P. \& PHAFF, H.J. 1956. Studies on the ecology of Drosophiza in the Yosemite region on California III. The Yeast flora on the natural breeding sites of some species of Drosophi 2a. Ecology, 37: 538-544.

DA CUNHA, A.B. 1951. Modification of the adaptative values of chro mosomal types in Drosophiza pseudoobscura by nutritional variables. Evolution, 5: 395-404.

DA CUNHA, A.B.; SheHAIA, A.M.I. \& de OLIVEIRA, W. 1957. A study on the diets and nutritional preferences of tropical species of Dro sophiza. Ecology, 38 (1): 98-106.

DOBZHANSKY, Th. \& PAVAN, C. 1950. Local and seasonal variation in relative frequencies of species of Drosophiza in Brazil. The Jour nal of Animal Ecology, 19(1): 1-14.

DUDGEON, E. 1954. Species differences in the utilization of wild yeast by Drosophiza. Univ. Texas Publ., 5422: 65-97.

FORTES, A.B. 1959. Geografia Física do Rio Grande do Sul. Ed. Livra ria do Globo. Porto Alegre.

FRANK, G. 1982. Estudo sobre flutuações nas populações de Drosophiza em Bento Gonçalves (RS) e aspectos cromossōmicos da Drosophiza angustibucca Duda. Dissertação de Bacharelado, Curso de Ciências Biolōgicas, ênfase em Genētica, UFRGS.

HEED, W.B. 1957. Ecological and distributional notes on the Droso phiza (Diptera) of El Salvador. In: Studies in the genetics of Drosophiza. Univ. Texas Publ., 5721: 62-78.

HOENIGSBERG, H.F.; PALOMINO, J.J.; CHIAPPE, C. ; ROJAS, G.G. \& CANAS, B.M. 1977. Population genetics in the american tropics. XI. Sea sonal and temporal variations in relative frequencies of species belonging to the wizzistoni group in Colombia. Oecologia (Berl.), 27: 295-304.

HOFMANN, P.R.P. 1982. Relações genētico-ambientais em Drosophiza in compta - uma espécie de ecologia restrita. Dissertação de Mestra do. Curso de Pós-Graduação em Genética, Universidade Federal do 
Rio Grande do Sul.

HUBBY, L. \& LEWONTIN, R.C. 1966. A molecular approach to the study of genetic heterozygosity in natural populations. I. The number of alleles at different loci in Drosophila pseudoobscura. Genetics, 54 : $577-594$.

IRGANG, B.E. 1980. A mata do Alto Uruguai, RS. Ciência e Cultura, $32(3): 323-324$.

JAENIKE, J. 1978. Ecological genetics in Drosophila athabasca. Its effect an local abundance. The American Naturalist, 112(984):287 -299 .

JOHNSON, F.M.; KANAPI, C.G.; RICHARDSON, R.H. \& LAKAI, R.K. 1966. Isozyme variabiliti in species of the genus Drosophila. I. A mul tiple allelic isozyme system in Drosophila busckit: inheritance and general considerations. Biochemical Genetics, 1: 35-40.

JOHNSTON, J.S. \& HEED, W.B. 1976. Dispersal of desert-adapted Droso phila: The Saguero-breeding D. nigropiracula. The American Natu list, $110(974): 629-651$.

KANESSHIRO, K.J.; CARSON, H.L.; CLAYTON, F.E. \& HEED, W.B. 1973. Ni che separation in a pair of homosequential Drosophiza species from the island of Hawaij. The American Naturalist, 107(958): $766-774$.

Mac ARTHUR, R.H. \& PIANKA, E.R. 1966. On optimal use of a patchy en vironment. Am. Nat., 100: 603-609.

MARQUES, E.K.; NAPP, M.; WINGE, H. \& CORDEIRO, A.R. 1966. A cornmeâl, soybean flour, wheat germ medium for Drosophila. Drosophila in form. Serv., 41: 187.

MATHER, W.B. 1956. The genus Drosophila (Diptera) in Eastern Queens land. II. Seasonal changes in a natural populàtion in 1952-1953. Austr. J. Zool., 4: 65-75.

MraK, E.M. \& PHAFF, H.J. 1948. Yeasts. Ass. Microbia1., 2: 1-46.(Ci tado por PAVAN, 1959).

PARSONS, P.A. 1975. The efect of temperature and humidity on the distribution patterns of Drosophila inornata in Victoria, Austra lia Environ. Entomol. 4: 961-964.

PARSONS, P.A. 1977. Larval reation to alcohol as an indicator of resource utilization differences between Drosophila melanogaster and D. simulans. Oecologia (Berl.), 30: 141-146.

PARSONS, P.A. \& STANLEY, S.M. 1981. Domesticated and widespread spe cies. In: The Genetics and Biology of Drosophita. Vol. 3 eds. M. Ashburner, H.L. Carson and J.N. Thompson Jr., Academic Press. p. 349-393.

PATTERSON, J.T. \& WAGNER, R.P. 1943. II. Geographical distribution of species of the genus Drosophiza in the United Stats and Mexi co. Studies in the Genetics of Drosophila. The Univ. Texas Publ., 
4313: $217-281$.

PAVAN, C. 1952. Relàções entre populações naturais de Drosophiza e

o meio ambiente. Tese. Faculdade de Filosofia, Ciências e Le tras. Universidade de são Paulo.

PAVAN, C. 1959. Relações entre populações naturais de Drosophiza e o meio ambiente. Tese apresentada ao concurso de Cátedra da Ca deira de Biologia Geral, Faculdade de Filosofia, Ciências e Le tras da USP em 1953. Boletim nọ 221, Biologia Geral nọ 11.

PETERSEN, J.A. 1960. Studies on the ecology of the genus Drosophiza.

I. Colection in two different life zones and seasonal variations in Rio Grande do Sul, Brazil. Rev. Bras. Biol., 20 (1): 3-16.

PHAFF, H.J.; MILLER, M.W.; RECCA, J.A.; SHIFRINE, M. \& MRAK, E.M. 1956. Studies on the ecology of Drosophiza in the Yosemite region of California. II. Yeasts found in the alimentary canal of Drosophi Za. Ecology, 37: 533-538.

PITTENDRIGH, C.S. 1958. Adaptation, natural selection and behavior. In: Behavior and Evolution. Anne Roe and G.G. Sempson (eds.), p. 390-416. University Press Yale, New Haven. (Citado por WINGE, $1971)$.

RAMBO, B.A. 1956. A fisionomia do Rio Grande do Sul. Vol. VI da sẹ rie "Jesuítas no Sul do Brasil". Selbach, P.A., 20 ed.

SAALFELD, K. 1979. Parāmetros ecolōgicos e genéticos de uma popula ção natural de Heliconius erato phyzzis (Lapidoptera: Nymphalidae). Tese de Mestrado - Departamento de Genētica, UFRGS.

SAAVEDRA, C.C.R. \& NAPP, M. 1983. Variação alozímica em populações naturais de Drosphila guaramunu do Rio Grande do Sul. (Comunica ção pessoal).

SENE, F.M.; VAL, F.C.; VILELA, C.R. \& PEREIRA, M.A.Q.R. 1980. Prel $\underline{i}$ minary data on the geografical distribution of Drosophila species within morphoclimatic domains of Brazil. Papéis Avulsos Zool.,33 (22): $315-326$.

TODA, M.J. 1973 b. Seasonal activity and microdistribution of dro sophilid flies in Misumai in Sapporo J. Fac. Sci. Hokkaido Univ. (VI - Z001), 18: 532-550.

TODA, M.J. \& KIMURA, M.T. 1978. Bionomics of Drosophilidae (Diptera) in Hokkaido I Scaptomyza palzida and Drosophiza nipponica. Ken tyû, Tockyo, 46(1): 83-98.

WINGE, H. 1971. Níveis de divergência evolutiva no grupo críptico da Drosophiza wizlistoni. Tese de Doutorado. Curso de Pós-Gradua ção em Genética da Universidade Federal do Rio Grande do Sul.

Recebido em setembro, 1985; aceito em novembro, 1985. 
